\title{
Smart Parking System Based on Embedded System and Sensor Network
}

\author{
Faiz Ibrahim Shaikh \\ Student, Department of \\ Computer Engineering, \\ JSPM'S Jaywantrao \\ Sawant College of \\ Engineering, \\ Pune, India
}

\author{
Pratik Nirnay Jadhav \\ Student, Department of \\ Computer Engineering, \\ JSPM'S Jaywantrao \\ Sawant College of \\ Engineering, \\ Pune, India
}

\author{
Saideep Pradeep \\ Bandarkar \\ Student, Department of \\ Computer Engineering, \\ JSPM'S Jaywantrao \\ Sawant College of \\ Engineering, \\ Pune, India
}

\author{
Omkar Pradip Kulkarni \\ Student, Department of \\ Computer Engineering, \\ JSPM'S Jaywantrao \\ Sawant College of Engineering, \\ Pune, India
}

\author{
Nikhilkumar B. Shardoor \\ Assistant Professor, \\ Department of Computer Engineering \\ JSPM'S Jayawantrao \\ Sawant College of Engineering \\ Pune, India
}

\begin{abstract}
Locating a parking space in central city areas, especially during the peak hours, is cumbersome for drivers. The issue arises from not having the knowledge of where the available spaces may be at the time, even if known, many vehicles may seek very limited parking spaces to cause severe traffic congestion. In this paper the design and implementation with a prototype of Reservation-based Smart Parking System (RSPS) that permits drivers to effectively locate and withhold the vacant parking spaces in mentioned. This system use cluster based algorithm which helps in periodically learning the parking status from the sensor networks deployed in parking spaces, the reservation service is influenced by the change of parking status. The drivers are allowed to access this said cyber-physical system with their personal communication devices. The system implemented is cost efficient smart parking system for multi-level parking facility using WSN (IR Sensor) and develop an android based application, by cluster based allocation method and performs automatic billing process. The system monitors the availability of idle parking slots and guides the vehicle to the nearest free slot. Cost is minimized by keeping the number of sensors low without sacrificing the reliability. Energy consumption of each mote is kept in check by allowing the systems to sleep periodically and by reducing their communication range. This system's reservation-based parking policy has the potential to smoothen the operations of parking systems, as well as mitigate traffic congestion caused by searching for parking.
\end{abstract}

\section{Keywords}

wireless sensor networks, internet of things, embeded system, reservation, resource alloaction, parking guidance and information.

\section{INTRODUCTION}

In today parking lots there are no standard system to check for parking spaces. The system heavily relies on human interaction with the physical space and entity. This leads to wastage of human manpower and also parking spaces at times. These parking lots are dependent on Human-toHuman Interaction (HHI) which is not efficient. Previously, various techniques have been proposed to overcome such problems like Short Messaging Services, Zigbee, RFID, etc.

Most of the time when users go to malls and commercial complex, they experience that there is a limited space for parking spots especially on prime hours. Hence, there is a desperate need of a robust parking system that will enable us to reserve the parking spots. For that it is necessary to build a centralized system to gather all the information on parking spots of malls, commercial complexes, and multilevel car parking systems. According to the International Organization of Motor Vehicle Manufacturers (OICA), the number of cars produced in 2014 alone stands at 60 million. With the explosive growth in automobiles, on-street parking will soon disappear, given the constraints on road space. Hence there is a desperate need of multilevel car parking and which should be smart also.

In urban areas, one-third of cars which have reached their destination and are circling around looking for a parking space thus leading to problems like pollution and traffic congestion. In a recent survey, researchers have found that in one year, car cruising for parking created the equivalent of 38 times trips around the world, burning 1.7 lakh litre of fuel and producing 730 tons of $\mathrm{CO} 2$. So, it is essential to control the air pollution using a robust parking system that will be used for the reservation of parking spot as well as parking spot allocation in on spot resource allocation scenario. Reservation can be made using multiplatform tools such as Android application, iOS application, Windows applications or even Web Portal allowing user to have a hassle free parking experience. Recommender system will help user find the right parking lot closest to his location.

With the help of recommender system predictability of nearest available slot becomes easier. User location data is accessed in the mobile platform to suggest best location for parking. The Google Map API uses user's geo-location and location of parking spot to determine the above. 


\section{SYSTEM ARCHITECTURE}

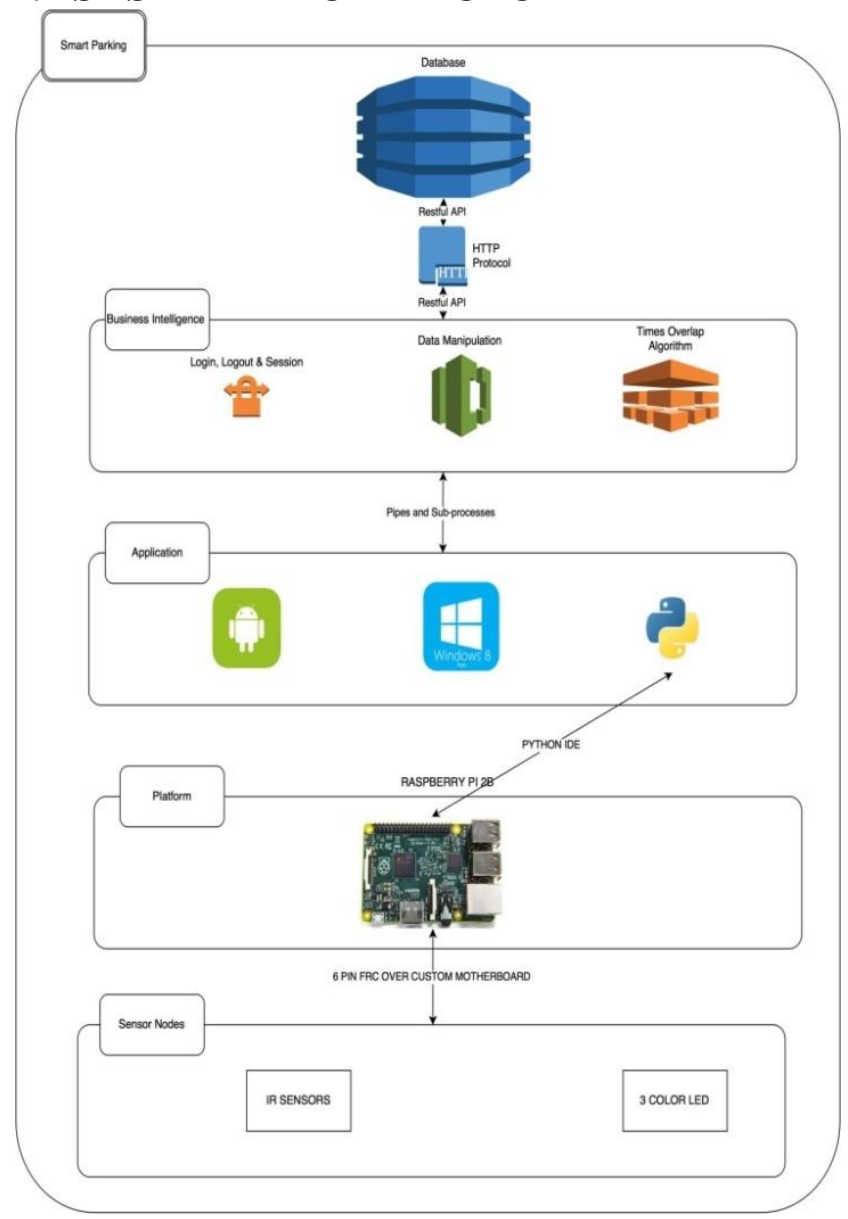

Figure 1. System Architecture

\subsection{Raspberry Pi}

The system is using Raspbian OS based on Debian OS running on raspberry pi $2 \mathrm{~B}$ model which has a configuration of ARM cortex-A7 quad core, $900 \mathrm{MHz}$ CPU . It also has 4 USB ports, 40 GPIO pins, Full HDMI port, Ethernet port, micro SD card slot. System has a 16GB SD card of class 10 which is used to mount Raspbian OS. System is designed in such a way that it will use Raspberry Pi as a web server. Raspberry Pi is mounted with a custom designed shield that is used to connect 4 IR sensors using FRC cable. Hardware system consists of 4 IR sensors which are connected to raspberry pi through FRC cables.

The system uses 24 GPIO pins to interact with IR sensors and tricolor LEDs. They are connected over a common VCC and GND.

\subsection{IR Sensors}

An infrared sensor is an electronic device which is used to sense light wavelength of its surroundings by either emitting or detecting infrared spectrum. It is also capable of measuring the heat being emitted by an object and detecting motion. LED will glow with respect to the IR sensor detection. Every IR sensor has a transmitter and a receiver. For every transmitter and receiver, there are total two registers and one more register is present to maintain voltage of LED that will show the status of parking slot to customers.
When the IR does not detect any object in front of it, it returns the value TRUE, meaning HIGH volt output from the sensor and FALSE for when a object is detected meaning LOW volt output.

\subsection{LED}

A tricolor LED has a cut section at either of its sides. These LED have 4 pins. The longest one is common cathode and the rest are represented as RGB.

\subsection{Parse}

Parse is an open source web server framework that allows the implementation of the back end server. It is suitable for implementation of a prototype for various projects. Parse works as a mediator between the Raspberry $\mathrm{Pi}$ and other Platform applications. Parse handles the NoSQL database behind the system. It also facilitates the use of push notifications through its services.

\subsection{Admin Dashboard}

Admin gets different kinds of data from various sources in JSON formats on its dashboard. Admin dashboard shows the activity logs, reserved parking slots, free parking spaces. It is also responsible for the parking spot allocation to different users. Algorithm will allocate the nearest free parking space to various customers who don't reserve their parking spaces.

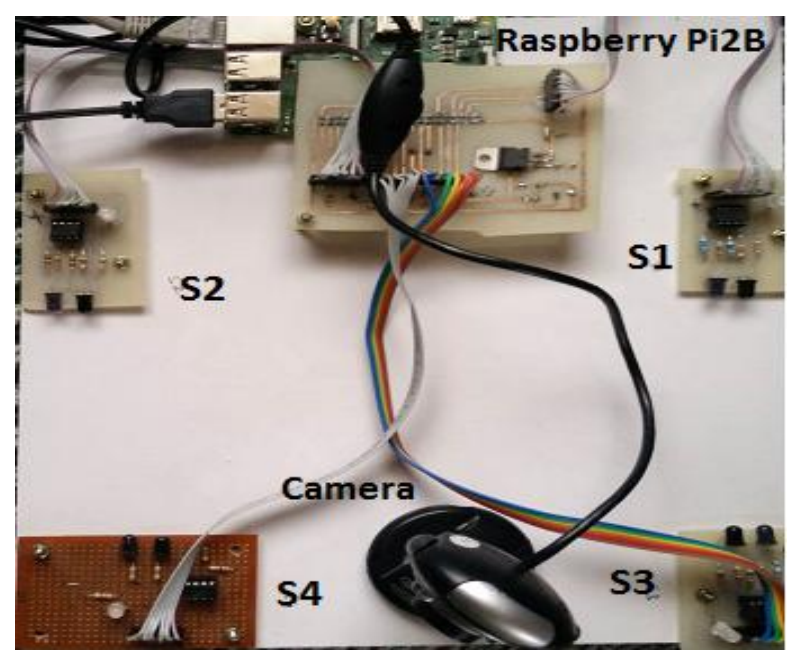

Figure 2. Hardware Architecture

\section{SYSTEM REALIZATION \& WORKING}

\subsection{Parking Space Detection:}

The system works on real time parking information based on which it makes reservation and on spot resource allocation of parking spaces for drivers. Several sensing technologies available to monitor real time parking status like ambient light sensor, Ultra sonic sound,etc. System works on IR led Sensors providing Energy efficiency with less power consumption.

IR led keeps transmitting infrared rays up to some range when some object is detected in the infrared range the IR waves hits the object and comes back at some angle the photo diode next to IR led detects that infrared rays reflected from the object.

Thus the object or the car standing in front of the IR sensors gets detected, providing us the status or availability of a 
particular parking space.

\subsection{V2I and I2V Communication}

For the need of utilization of resources a reliable and effective two way communication is essential between the vehicle and the allocation center (infrastructure).

In this system V2I communication involves drivers sending their parking request providing driver information, status confirming reservation and utilization of resources to the system. In I2V communication the system reserves the space for user and the application shows the driving directions to the reserved parking space with the help of a directed map, the map contains the images of the path allocated to the driver by the system.

Both I2V and V2I communication are implemented through an Android application \& other Windows or Web application.

\subsection{Reservation Guarantee}

In case of an efficient parking system the resources allocated to a particular user must not be designated to any other user. To successfully imply this idea, QR Code Generation technology is applied.

The system does QR code verification at the entrance of the parking lot with the help of a web camera which is placed on the system. User with its application reserves a parking space, QR code gets generated on the reservation which provides unique "object id" for every user on every transaction. This generated QR code should be presented in front of the camera. The System scans and detects the unique object ID and then verifies the ID from the database.

\subsection{Optimal Allocation}

The most powerful feature of the system is that it determines the nearest or closest parking space for the user with the help of globally optimized list.

The Admin dashboard fires the query on the database through the application in such a way that it returns only the unreserved resources which are globally optimized. On the acquired list linear search is performed and it gives the closest parking slot from the list which also the first element of the optimized list[O(1)].This helps in optimal allocation of the resources through the system.

\subsection{Backend}

For the efficient working and communication of the server, application and database backend devices use server side languages to build an application and data storage tools to find change or save data and serve it back to the user.

The system uses NoSQL database management system which contains unstructured data. NoSQL also provides storing of images and videos. It also is scalable, instead of using tables NoSQL stores data in the form of objects and $\mathrm{key} / \mathrm{value}$ pairs tuples.

JSON (Java Script Object Notation) it is a light weight format used for data interchanging. It is used as a web service response. Instead of using XML as a primary data format for the transfer of data JSON is more preferred format due to its light weight property.

RESTful API (Representational State Transfer Application Programming Interface) is used to ease development and simplify client adoption it does not follow a prescribed standard except for HTTP. System uses four important methods viz POST, GET, PUT, DELETE.

- POST: POST is utilize to "create" new resources especially used to create subordinate resources means POST to the parent. On creation, return HTTP status 201.

- GET: GET method is used to "read" or "retrieve" resource. It returns representation in JSON and HTTP response code 200 and in case of error it returns 404 .

- PUT: PUT is utilize for "update" the resources it can also be used to create resource when the resource id is chosen by client instead of server.

- DELETE: It is used for "deleting" the resource. On deletion it returns HTTP status 200 with response body. It can also return HTTP status 204 with no response body.

\subsection{Sensors}

Sensors are the devices which senses from the physical world and stimuli on the environment. The system uses IR sensors to detect the vehicle above it.

Sensors respond to system to the availability of the vehicle above the IR sensors. If the vehicle is present above the IR sensor, it will return value FALSE and the voltage goes LOW, If the vehicle is not present above the IR sensor, it will return value TRUE and the voltage remains HIGH.

The parking space is reserved but the vehicle is yet to arrive, the LED glows RED. In another case vehicle is at the entrance of parking lot then LED glows BLUE, to notify the driver the parking slot allocated to him. When a vehicle has already occupied a parking slot the led will glow GREEN. The GREEN LED is used as a positive indicator to showcase proper functioning of IR Sensors.

\subsection{Data Analysis}

\section{- Nearest resource allocation}

Admin fires the query to get the globally optimized list. The list contains only the unreserved free parking spaces available in the parking lot. The list is natively optimized so, the first element in the list is the nearest of all.

\section{- QR scanner:}

Every time when user reserves a parking slot for him, a QR code is generated along with the payment receipt with the help of unique object id. While entering in the parking lot, user first has to scan his unique QR code. For that user has to place his QR code i.e. smart phone in front of the webcam. The webcam will scan the QR code and object id along with the name of reserved parking slot will get displayed. Admin dashboard updates the logs and the event gets generate by glowing the LED light BLUE. User already has a directed map showing the exact location of parking space. Only after a successful authentication via $\mathrm{QR}$ code, user is allowed to enter into the parking area.

\section{- System Logs:}

System $\log$ is produced on the admin dashboard showing all the activities which are done in the 
past. It includes username, vehicle number, object id for every transaction, slot number allocated to user, time of arrival and departure, date of reservation etc.

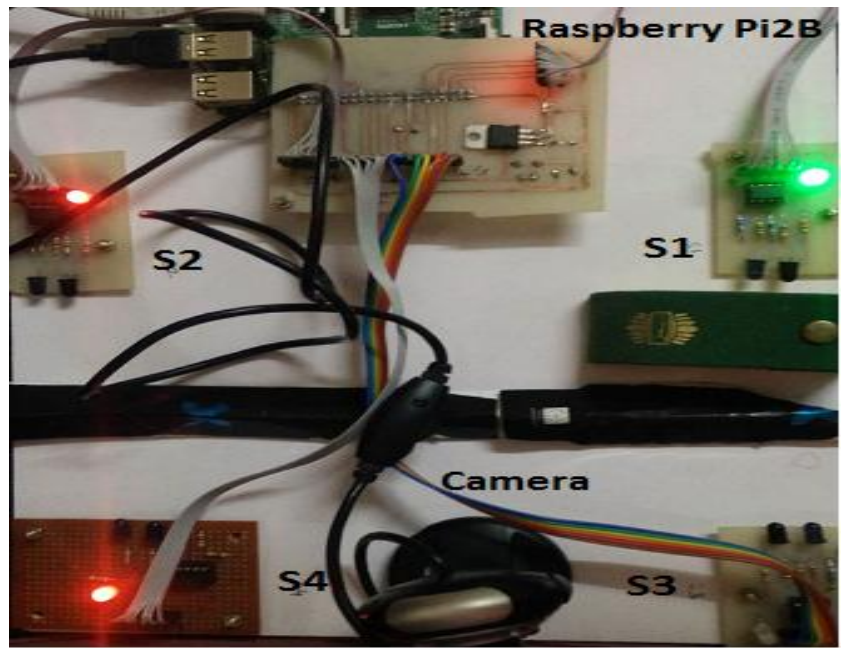

Figure 3. Working state(Slot S2 \& S4 Reserved and S1 occupied)

\subsection{Platform}

An Android application is created using Android studio. The android applications are developed using the JAVA code. Using the JAVA compiler the source files are converted to JAVA class files. The .dex file and the resources of an android application are packed into an apk (Android Package) file. The resulting .apk file contains all data to run the Android application and can be deployed to an Android device using $a d b$ tool.

The Windows Application is made using Visual Studio 2012. Visual Studio allows us to create windows forms and windows runnable applications. This application has its minimum requirements according to the safety standards of .Net Framework. This platform generates a setup file for the "Smart Parking" and allows us to run the .exe for creation of account to reserving parking.

Using the slot allocation method these application are developed for the "Smart parking". Slot reservation can be done using the slot allocation method. The request is updated in the server and forwards it to parking area. Platform allows user to enter arrival and departure time of the parking. Both platform application ensures that reservation of parking spaces is allowed maximum two days before the actual reservation date. The reserved spot will be available again only after three hours of departure time of previous reserved vehicle, maintaining a buffer for consecutive reservation of same parking spot.

\section{MATHEMATICALNMODELBAND SIMULATION RESULTS:} $\mathrm{S}=\{\mathrm{C}, \mathrm{Fme}, \mathrm{S}, \mathrm{F}\}$

Let,

$\mathrm{C}=\{\mathrm{FL}, \mathrm{OL}, \mathrm{RL}\}$

Where,

- $\quad$ FL be set of Free Lots $\{$ I1,I2,I3,In $\}$

- $\quad$ OL be set of Occupied Lots $\{$ I1,I2,I3,In $\}$
- $\quad \mathrm{RL}$ be set of Reserved Lots $\{\mathrm{J} 1, \mathrm{~J} 2, \mathrm{~J} 3, \mathrm{Jn}\}$

$\mathrm{Fme}=\{\mathrm{LS}, \mathrm{RS}, \mathrm{Lo}, \mathrm{Rr}, \mathrm{Qr}, \mathrm{OSr}\}$

Where,

- Let LS be the LED Light Status \{BLUE(B), $\operatorname{GREEN}(\mathrm{G}), \operatorname{RED}(\mathrm{R})\}$

Where,

- BLUE (B) is currently assigned slot.

- $\operatorname{GREEN}(\mathrm{G})$ is currently occupied Lot.

- $\quad \mathrm{RED}(\mathrm{R})$ is currently reserved Lot.

- Let RS be Reservation Status \{NOT RESERVED (nRS),RESERVED(RS)\}

Where,

- NOT RESERVED (nRS) is Reservation not done.

- RESERVED (RS) is Reservation done

- $\quad$ Login (Lo) is User Login Function

- Reservation Request (Rr) is Function to reserve specific lot

- $\mathrm{Qr}$ is Generated Qr code after successful Reservation

- On Spot Request (OSr) is to request nearest free parking lot without registration

Let $\mathrm{S}$ be Set of successful cases

$\mathrm{S}=\{\mathrm{Rp}, \mathrm{OSp}\}$

Where,

- $\quad$ Park (Rp) is successful occupied lot

- On Spot Park (OSp) is successful occupied lot $\mathrm{F}=$ Failure Cases where Qr Code was not generated, Parking lot was not reserved

\section{MOBILE BACK END AS A SERVICE (MBaaS)}

It is a model for providing web and mobile application developers with a way to link their applications to back end server which also can be used as a cloud storage. APIs exposed by back end applications MBaaS also provides features such as user management, push notifications, analysis of performance measures and integration with social networking services, file storage, geo-location and ability to store custom objects. These services are provided via the use of custom software development kits (SDKs) and application programming interfaces (APIs). Backend as a Service eliminates the need for developers to construct their own servers. MBaaS providers offer developers a ready-made, customizable back end that's already outfitted with common back-end features. As a result, proponents of the approach say it shifts developers' focus away from time-consuming, complex server end development, and gives them more time to invest in front-end work such as UI/UX. The scalability of mobile apps, which is a huge pain-point for mobile app developers, is also handled by MBaaS providers; apps are scaled automatically, in the cloud, according to variations in volume of users and traffic.

6. PROTOTYPE IMPLEMENTATION:

The proposed system has been partially implemented and tested in lab prototype. Client devices have been connected via TCP/IP protocol to a parking database. The latter is updated in real-time with the status of parking lots. Two kinds of client applications have been considered for parking lots monitoring: mobile device application, for phones and 
tablets, and desktop application for laptops and desktop computers.

\subsection{Hardware Prototype}

Hardware prototype contains 4 IR sensors as of now that will represent the 4 parking spaces as shown in fig.2.According to fig1, these IR sensors are connected through wires to Raspberry Pi which will also act as a server. A webcam is also attached to Raspberry Pi to scan the QR code for the authentication of the driver at entry point. Internet is provided to Raspberry Pi via LAN connection to send the real time data to admin dashboard.

\subsection{Android Application Prototype}

Android application contains only one parking area. That parking area contains only 4 parking slots viz. S1, S2, S3, and S4 which are present on the hardware. Fig.4 shows the layout of parking slots as well as 3 legends to depict whether the particular slot is "Available", "Unavailable/Reserved"," User selected".

Fig. 5 shows the details of the reservation. It contains name of parking area, name of parking slot, arrival time, departure time, date and duration. User must have to enter his vehicle number to uniquely identify his vehicle in case a user has multiple vehicles.

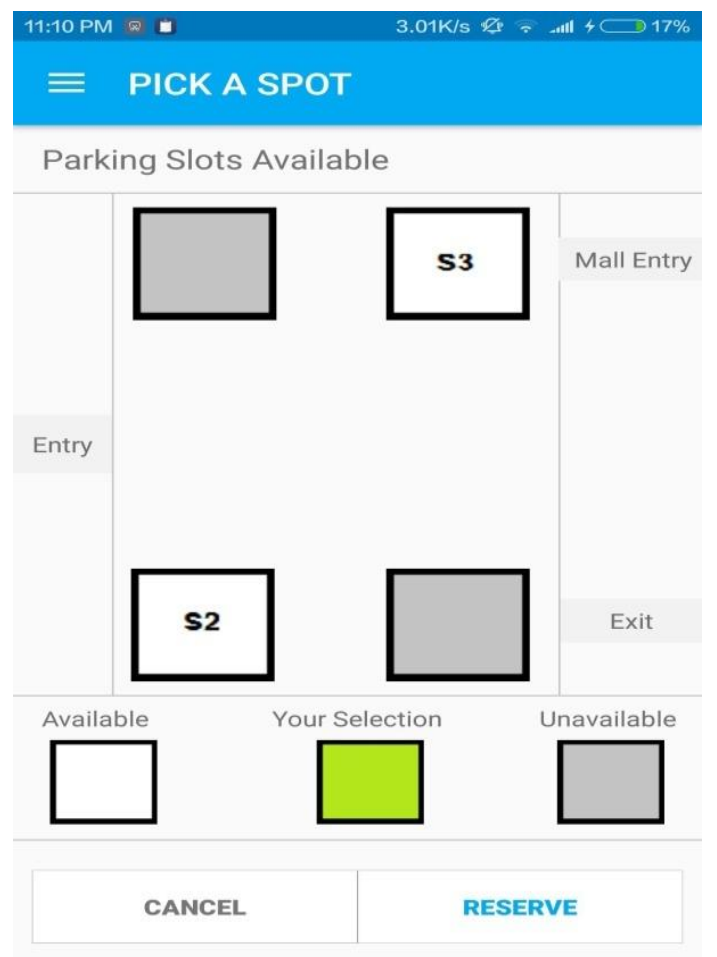

Figure 4. Android app prototype shows S1 and S4 are unavailable or reserved and only S2, S3 are available.

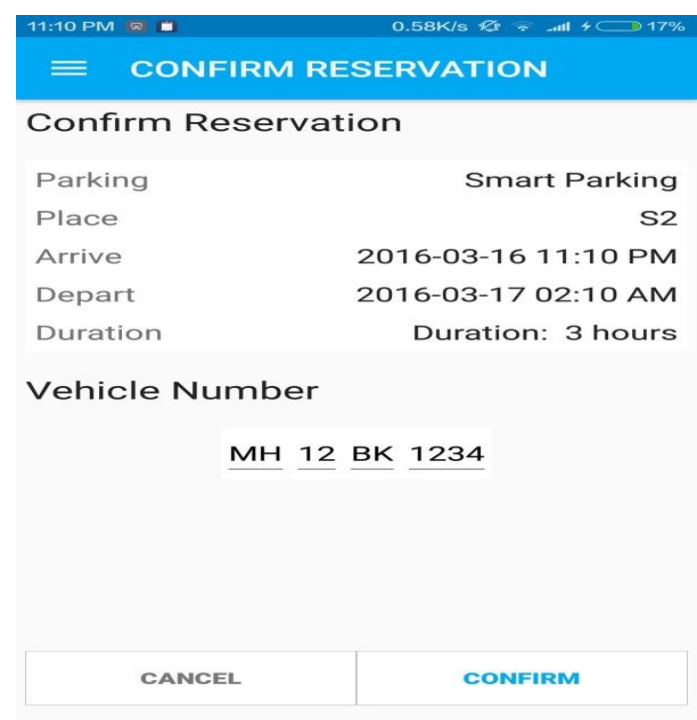

Figure 5. Android app prototype shows the details of reservation before confirming the slot

\subsection{Windows Desktop Application:}

On windows desktop application user has to enter arrival time, departure time, date, and vehicle number. Then windows application will show the map and layout of all parking lots which are similar to the android application. It will also show the available parking slots so that user can enter the desired resource very easily. After clicking on the "Book" button user will be greeted with a pop up window showing the QR code and other relevant information about reservation. 


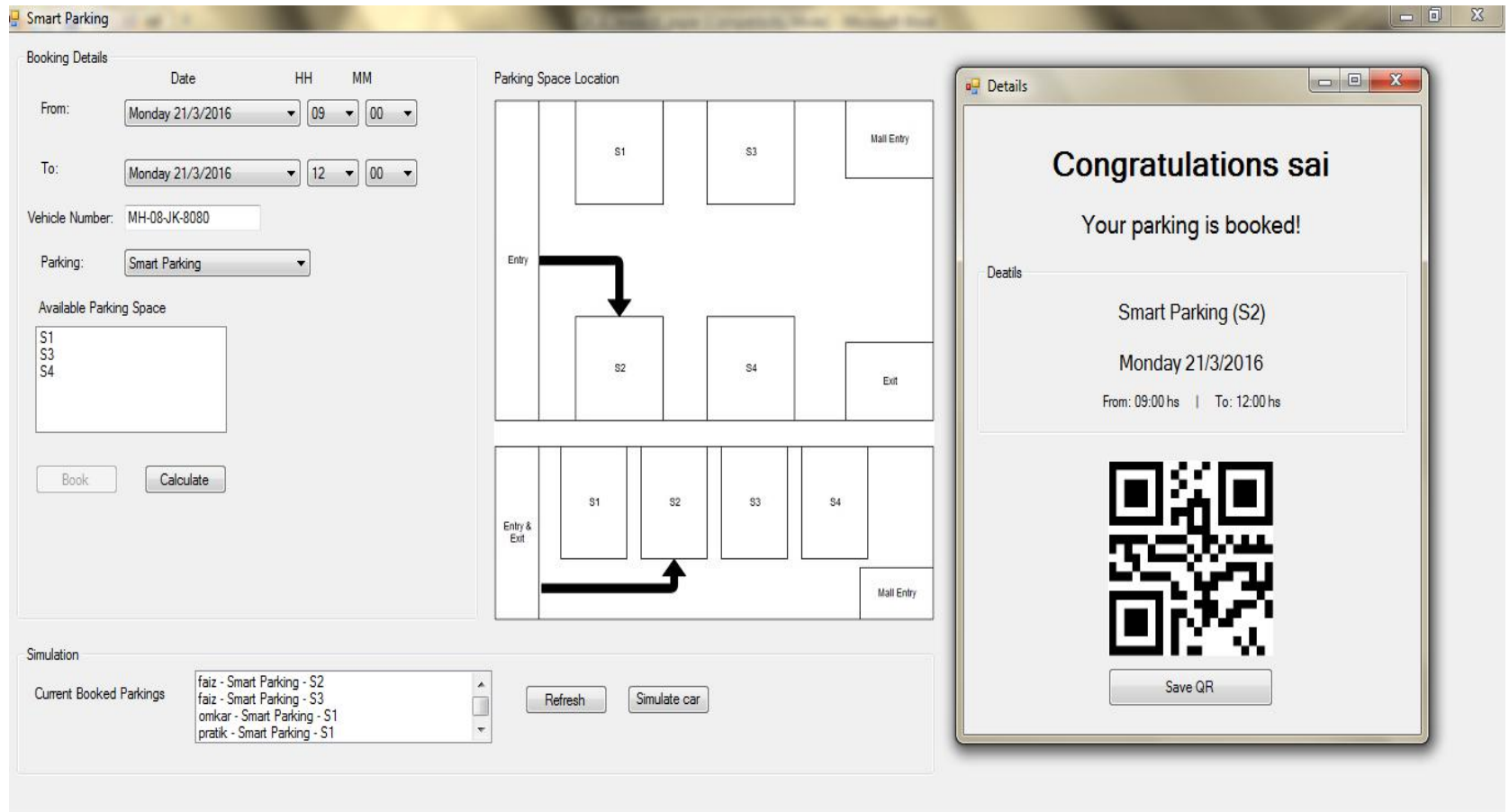

Figure 6. Windows application showing the reservation has been made for parking slot S2

\section{PERFORMANCE EVALUATION}

The authors conducted a number of experiments and simulations to quantitatively investigate the impact of the proposed framework in terms of engineering and economic efficiency. Three classes of performance metrics are considered. The first tab is to check which database class the query is being fired upon. The 2nd tab suggests which type of query the application is requesting. 3rd tab gives the total number of counts and fourth calculated the slow percentage of query. The "Median" tab gives the avg. time required to give the output of the query which is no longer than 7 milliseconds which is much less that SQL query which takes

\begin{tabular}{|c|c|c|c|c|c|c|c|}
\hline Class: & Normalized Query $\bullet$ & Count & sions e & Tineouts & Scanned (AvB)e & Median (es) & Pgo (cos) $\bullet$ \\
\hline Parkings & where: \{\} & 49 & 0.05 & 8.05 & 1.8 & 5.27 & 15.278 \\
\hline _User & where : $[$ usernase: : .. I & 25 & $\theta .0 \mathrm{x}$ & 0.00 & 1.0 & 4.557 & 15.200 \\
\hline Booked & where : $[$ user: $\ldots]$ & 15 & 6.675 & 8.05 & 1.0 & 5.751 & 112.218 \\
\hline Parkings & where : $\{$ name: $\ldots\}$. & 11 & 9.094 & 0.05 & 1.0 & 5.155 & 8.525 \\
\hline Booked & where: $\{$ parking: $\ldots$ ] & 11 & 0.05 & 0.08 & 3.0 & 5.669 & 7.054 \\
\hline Booked & where: $[\mathrm{s}$ & 11 & 0.08 & 0.08 & $2 . \theta$ & 5.008 & 5.649 \\
\hline Booked & where : $\{$ parking: $\ldots$, place: $\ldots$. $]$ & 7 & $\theta, \theta \mathrm{s}$ & $0.0 \mathrm{~s}$ & $\theta . \theta$ & 4.497 & 624.19 \\
\hline -Ro1e & where: : [\} & 6 & 0.05 & e.0s & 9.0 & 4.045 & 5.977 \\
\hline Session & where: [ $]$ & 2 & 0.09 & e.es & $9 . \theta$ & 6.773 & 6.773 \\
\hline
\end{tabular}

Figure 7. Query Analysis over a median time

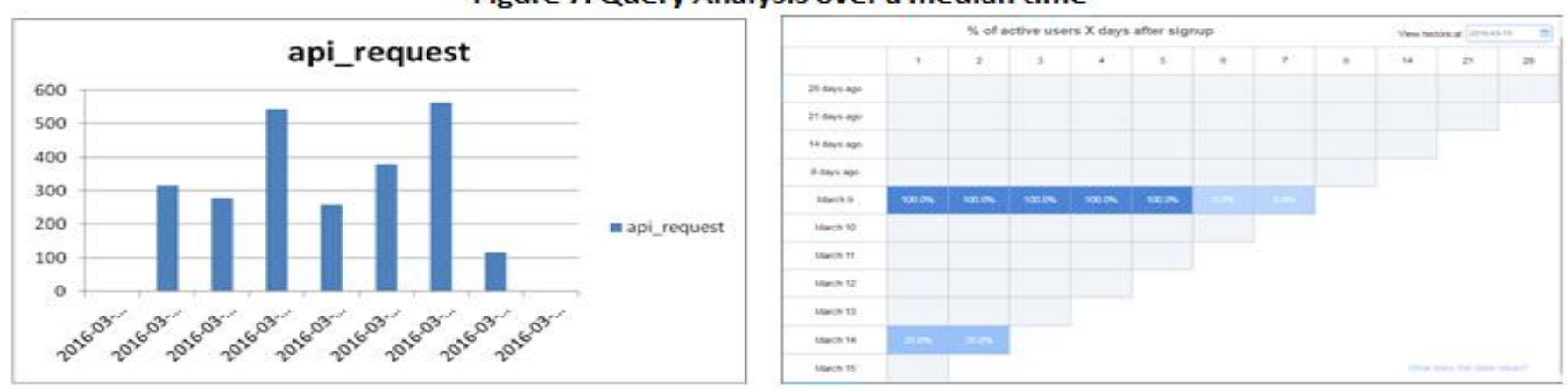

Figure 8. API Request and Data retention over a period of time 
appx. $5 \mathrm{sec}$ on an average. The last tab gives the maximum time required for a query to fetch data over all its performance.

\section{COMPARISON}

The figure below gives a comparison of the Smart Parking system which allows reservations over the various system such as
1. Blind Search
2. Parking Information Sharing
3. Buffered PIS
4. Smart Parking

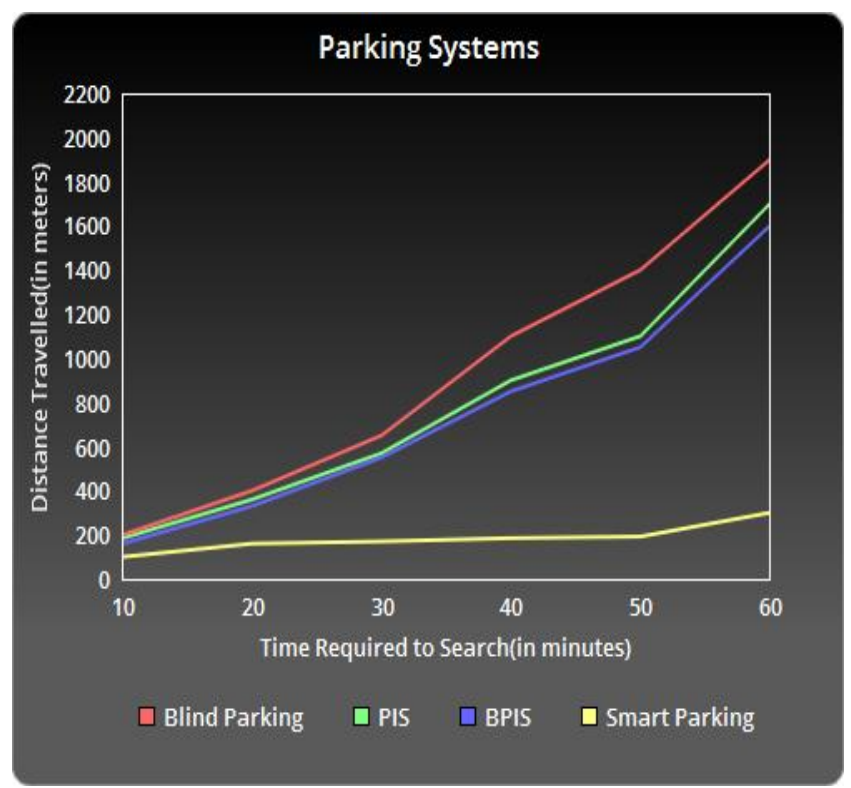

Figure 9. Comparison of Smart Parking with other parking systems

Over a given period of time the Smart Parking System proves efficient by providing real time data and facility to book the parking.

\section{CONCLUSION AND FUTURE SCOPE}

Various system proposed by different authors helps us to cogently in reserving and also annihilating the need of searching for parking spaces in private parking lots. Researchers have acquired the systems which dynamically arrange the scheme for different drivers as per their requirement, based on the real-time parking information. Thus, this concludes that the paper simplifies the context for the researchers for innovating various techniques to administrate and solve the problems faced by drivers on day to day basis.

In future, the system can be extended which is not only specific to a private parking like Malls, Company parking, etc. but also can be implemented over various multiple platforms such as public parking also extending the feature by giving parking information based on cost in real time. This will make the management of the parking spaces more efficient, by purging the need of human labor.

\section{ACKNOWLEDGEMENTS}

This project is partially funded by SHREE ENTERPRISES.
It is also co-funded by the project members under the academic curriculum of SAVTRIBAI PHULE UNIVERSITY. We are grateful to our guide Prof. Nikhilkumar B. Shardoor Assistant professor, Computer Department, JSPM's Jaywantrao Sawant College of Engineering for helping us in preparation of this paper and his valuable suggestions and encouragement.

\section{REFERENCES}

[1] Faiz Shaikh, Nikhilkumar B.S., Omkar Kulkarni, Pratik Jadhav, Saideep Bandarkar "A Survey on "Smart Parking" System", IJIRSET, 10.15680/IJIRSET.2015.0410088.

[2] ZigBee/IEEE 802.15.4 Summary, "Sinem Coleri Ergen,”tech. rep., EECS Berkely, September 2004.

[3] Yanfeng Geng, Student Member, IEEE, and Christos G. Cassandras, Fellow, IEEE "New Smart Parking System Based on Resource Allocation and Reservations", IEEE Transactions on intelligent transportation systems, VOL. 14, NO. 3, September 2013.

[4] P.Dharma Reddy, A. Rajeshwar Rao, Dr. Syed Musthak Ahmed, "An Intelligent Parking Guidance and Information System by using image processing technique”, IJARCCE, Vol. 2, Issue 10, October 2013.

[5] ManjushaPatil, Vasant N. Bhonge "Wireless Sensor Network and RFID for Smart Parking System" International Journal of Emerging Technology and Advanced Engineering Website: www.ijetae.com (ISSN 2250-2459, ISO 9001:2008 Certified Journal, Volume 3, Issue 4,April 2013)

[6] M. M. Rashid, A. Musa, M. Ataur Rahman, and N. Farahana, A. Farhana, "Automatic Parking Management System and Parking Fee Collection Based on Number Plate Recognition.”, International Journal of Machine Learning and Computing, Vol. 2, No. 2, April 2012,Published 2014.

[7] Hilal Al-Kharusi, Ibrahim Al-Bahadly, "Intelligent Parking Management System Based on Image Processing", World Journal of Engineering and Technology, 2014, 2, 55-67.

[8] Harmeet Singh, Chetan Anand, Vinay Kumar, Ankit Sharma, "Automated Parking System With Bluetooth Access", International Journal Of Engineering And Computer Science ISSN:2319-7242,Volume 3 Issue 5, May 2014, Page No. 5773-5775

[9] El Mouatezbillah Karbab, Djamel Djenouri, Sahar Boulkaboul, Antoine Bagula, CERIST Research Center, Algiers, Algeria University of the Western Cape, Cape town, South Africa ,"Car Park Management with Networked Wireless Sensors and Active RFID”',978-14799-8802-0/15 @2015 IEEE

[10] Andre Barroso, Jonathan Benson, Tina Murphy, UtzRoedig, Cormac Sreenan, John Barton, Stephen Bellis, Brendan Flynn, and Kieran Delaney, "The DSYS25 Sensor Platform," tech. rep., University College, Cork, Ireland and National Microelectronic Research Center, Cork, Ireland,2004.

[11] Graph data from: Hongwei Wang, Wenbo He“A Reservation based Smart Parking System”, 2011 IEEE Conference, 10.1109/INFCOMW.2011.5928901. 